\title{
Hypermethylation-mediated silencing of NDRG4 promotes pancreatic ductal adenocarcinoma by regulating mitochondrial function
}

\author{
Hao-Hong Shi ${ }^{1}$, Hai-E Liu ${ }^{1}$ E Xing-Jing Luo ${ }^{1,2, *}$ \\ ${ }^{1}$ Department of Anesthesia, Children's Hospital of Fudan University, Shanghai 201102, ${ }^{2}$ Department of Anesthesia, Anhui Provincial \\ Children's Hospital, Hefei, Anhui 230022, China
}

\begin{abstract}
The N-myc downstream regulated gene (NDRG) family members are dysregulated in several tumors. Functionally, NDRGs play an important role in the malignant progression of cancer cells. However, little is known about the potential implications of NDRG4 in pancreatic ductal adenocarcinoma (PDAC). The aim of the current study was to elucidate the expression pattern of NDRG4 in PDAC and evaluate its potential cellular biological effects. Here, we firstly report that epigenetic-mediated silencing of NDRG4 promotes PDAC by regulating mitochondrial function. Data mining demonstrated that NDRG4 was significantly down-regulated in PDAC tissues and cells. PDAC patients with low NDRG4 expression showed poor prognosis. Epigenetic regulation by DNA methylation was closely associated with NDRG4 down-regulation. NDRG4 overexpression dramatically suppressed PDAC cell growth and metastasis. Further functional analysis demonstrated that up-regulated NDRG4 in SW1990 and Canpan1 cells resulted in attenuated mitochondrial function, including reduced ATP production, decreased mitochondrial membrane potential, and increased fragmented mitochondria. However, opposite results were obtained for HPNE cells with NDRG4 knockdown. These results indicate that hypermethylation-driven silencing of NDRG4 can promote PDAC by regulating mitochondrial function and that NDRG4 could be as a potential biomarker for PDAC patients. [BMB Reports 2020; 53(12): 658-663]
\end{abstract}

\section{INTRODUCTION}

Pancreatic ductal adenocarcinoma (PDAC) is one of the most malignant tumors with asymptomatic, rapid disease progression and poor prognosis (1). The vast majority of patients with PDAC

*Corresponding author. Tel: +86-021-64931990; Fax: +86-02164931990; E-mail: 11231240005@fudan.edu.cn

https://doi.org/10.5483/BMBRep.2020.53.12.168

Received 5 August 2020, Revised 3 September 2020, Accepted 13 October 2020

Keywords: Methylation, Mitochondrial function, NDRG4, PDAC are diagnosed in the advanced stage with a 5 -year overall survival rate of less than $6 \%$. Thus, PDAC is internationally called the "king of cancer" (2). Unfortunately, even with the advent of diverse detection technologies and advanced treatment methods, the incidence and mortality rate of PDAC are still on the rise year by year $(3,4)$. Due to the lack of in-depth understanding of molecular characteristics of malignant development, comprehensive and in-depth research studies are urgently needed to better prevent and treat PDAC.

$\mathrm{N}-\mathrm{Myc}$ downstream-regulated gene 4 (NDRG4) is a member of the NDRG family proteins (NDRG1-4) known to share 57\%$65 \%$ amino acid sequence identities (5). It is involved in cell proliferation, differentiation, development, and stress (6). NDRG4, the latest identified one, was initially found to be specifically expressed in the brain and heart $(7,8)$. In zebrafish, NDRG4 could maintain physiological levels of brain-derived neurotrophic factor (BDNF) to protect against neurological deficits for proper neurite outgrowth and neural functions $(7,9,10)$. In brains of patients with Alzheimer's disease, NDRG4 shows reduced expression. It is involved in neuronal degeneration (5). Besides, NDRG4 is involved in the regulation of normal cardiac morphogenesis in mouse and zebrafish. NDRG4 deficiency can lead to poor contractility and a decreased heart rate $(9,11)$.

Recently, it has been found that NDRG4 exerts various roles in human malignancies. In glioblastoma, NDRG4 plays an oncogene role. It is critical to the survival of astrocytes (12). In malignant meningioma, NDRG4 also plays an oncogene role by inhibiting p53 expression to suppress cell apoptosis (13). However, current studies in other tumors have found that NDRG4 works as a tumor suppressor. This inverse effect may indicate that NDRG4 expression has tissue-specific function in different types of cancer. In gastric cancer, significantly decreased expression levels of NDRG4 protein and mRNA are more likely to lead to poor prognosis (14). In colon and breast cancers, NDRG4 expression is decreased during the carcinogenesis process. Favorable outcome has been found in patients with higher NDRG4 expression $(15,16)$. These results indicate that NDRG4 might be a tumor suppressor in carcinogenesis. Interestingly, NDRG4 promoter methylation had been found in gastric cancer, colorectal cancer, and breast cancer, resulting in decreased NDRG4

ISSN: 1976-670X (electronic edition)

Copyright (C) 2020 by the The Korean Society for Biochemistry and Molecular Biology

(c) This is an open-access article distributed under the terms of the Creative Commons Attribution Non-Commercial License (http://creativecommons.org/licenses/by-nc/4.0) which permits unrestricted non-commercial use, distribution, and reproduction in any medium, provided the original work is properly cited. 
expression $(15,17,18)$. The expression pattern and regulatory mechanism of PDAC remain unclear.

So far, molecular characterization of NDRG4 and the role of this protein in PDAC have not been reported yet. In the present study, we found that NDRG4 was down-regulated in tumor tissues and positively associated with prognosis. Furthermore, our results demonstrated that NDRG4 was epigenetically silenced by DNA promoter hyper-methylation at cell level. Finally, we investigated the potential tumor suppressor function of NDRG4 by measuring cell proliferation, motility, and mitochondrial function in PDAC cells with stably overexpression of NDRG4.

\section{RESULTS}

\section{Abnormal expression of NDRG4 is correlated with PDAC progression}

Some studies have been performed to explore the function and mechanism of NDRG family members in multiple malignant cancers (6). To better illuminate the role of aberrant expression of NDRG family member in the prognosis of PDAC, data mining was carried out according to cross results of online analysis between Kaplan-Meier plotter (http://kmplot.com/analysis/) and oncolnc (http://www.oncolnc.org). Both online analyses revealed a statistically significant association between overall survival (OS) and expression of NDRG4 mRNA in PDAC patients, while only one online analysis showed a statistically significant association between NDRG2 or NDRG3 expression and OS (Fig. 1A). As shown in Fig. 1B, poor outcome based on median survival time (16.6 vs. 23.03 months) was found for PDAC patients with lower NDRG4 expression (log-rank $P=0.035$ ) analyzed by Kaplan-Meier plotter. Similar result was found for TCGA dataset analyzed by oncolnc (Fig. $1 C$, log-rank $P=0.0158$ ) when mRNA expression median was set as the cutoff. To unveil the expression pattern of NDRG4 in PDAC, the following analysis was performed with two GEO datasets (GSE28735 and GSE62452), including matched tumor and corresponding non-tumor tissues. Results showed significantly lower expression of NDRG4 in cancers than in paired adjacent tissues (Fig. $1 \mathrm{D}$ and $1 \mathrm{E}$ ). In addition, western blotting showed down-regulated NDRG4 protein in PDAC cells (PANC1, Canpan1, SW1990, and ASPC1) than in normal human pancreatic duct cells (HPNE) (Fig. $1 \mathrm{~F}$ ). These data suggest that down-regulated NDRG4 is implicated in the malignant transformation of PDAC.

\section{NDRG4 is epigenetically silenced by promoter DNA methylation}

The above results showed that NDRG4 was significantly downregulated in PDAC. However, the regulatory reason for the downregulation of NDRG4 expression in PDAC remains unclear. DNA methylation is a fundamental epigenetic modification to regulate gene expression. The genomic structure of NDRG4 with online analytical tools (https://www.ncbi.nlm.nih.gov/gene/ and http://www.urogene.org/methprimer/) showed a dense CpG
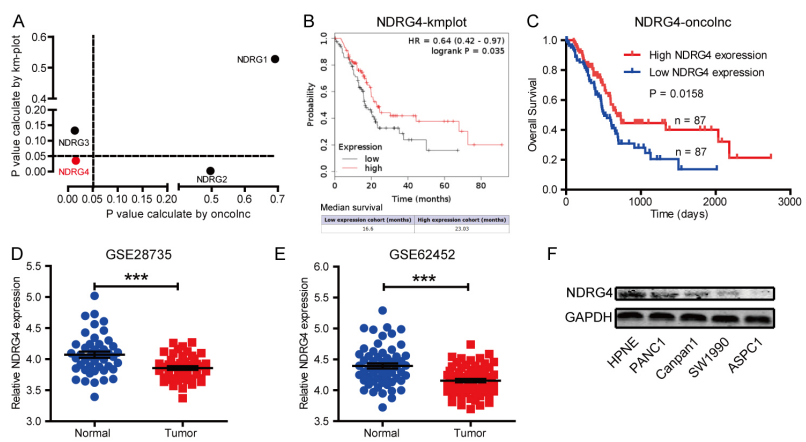

$\mathrm{F}$

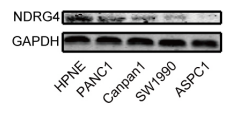

Fig. 1. Down-regulated NDRG4 is closely associated with poor PDAC patient survival. (A) The P-value distribution of NDRG family members' expression within PDAC patients' prognosis was analyzed with Kaplan Meier plotter (http://kmplot.com/analysis/) and oncolnc (http:// www.oncolnc.org/). (B) Kaplan-Meier plotter showed poor OS and less median survival time of PDAC patients with low NDRG4 expression (log-rank $\mathrm{P}=0.035, \mathrm{HR}=0.64$ ). (C) On-line analysis of Kaplan-Meier OS curves using TCGA dataset of PDAC patients by oncolnc. Patients were grouped on the basis of NDRG4 median expression value. (log-rank $\mathrm{P}=0.0158$ ). ( $\mathrm{D}$ and $\mathrm{E}$ ) NDGR4 expression levels were analyzed in tumors and adjacent tissues samples using two independent cohorts (D, GSE28735; E, GSE62452); Student's t-test, $* * * P<0.001$. (F) NDGR4 expression levels in PDAC cell lines and nonmalignant hTERT-HPNE (HPNE) cells were determined by immunoblotting.

island near the transcription initiation site (Supplementary Fig. 1). To investigate whether promoter methylation was associated with inhibition of gene expression, two PDAC cell lines (SW1990 and Canpan1) and a normal human pancreatic duct cell line (HPNE) were treated with gradient concentrations of DNA methylation inhibitor 5-aza-2'-deoxycytidine (DAC). Results showed that expression levels of NDRG4 in all PDAC cells, but not in HPNE cells, were significantly increased after treatment with DAC (Fig. 2A). To investigate the pattern of CpG island methylation in the NDRG4 promoter, bisulfite sequencing PCR (BSP) was performed for SW1990, Canpan1, and HPNE cells. Results revealed that SW1990 and Canpan1 cells showed almost complete methylation, whereas HPNE cells showed almost no methylated on CpGs (Fig. 2B) (region -151 to +10 relative to the transcription start site). Taken together, these data above suggest that NDRG4 is epigenetically silenced by promoter DNA methylation in PDAC.

\section{NDRG4 overexpression inhibits malignant properties of tumor cells}

To explore the role of NDRG4 in PDAC progression, SW1990 and Canpan 1 were transfected by lentivirus to upregulate NDRG4 expression. Meanwhile, NDRG4 knockdown in HPNE cells was achieved by treatment with siRNA (Fig. 4B) CCK8 assay showed that NDRG4 overexpression significantly inhibited viabilities of SW1990 (Fig. 3A) and Canpan1 (Fig. 3B) cells compared to control cells. The same results were obtained for clone formation (Fig. 3D and 3E). Nevertheless, opposite results were 


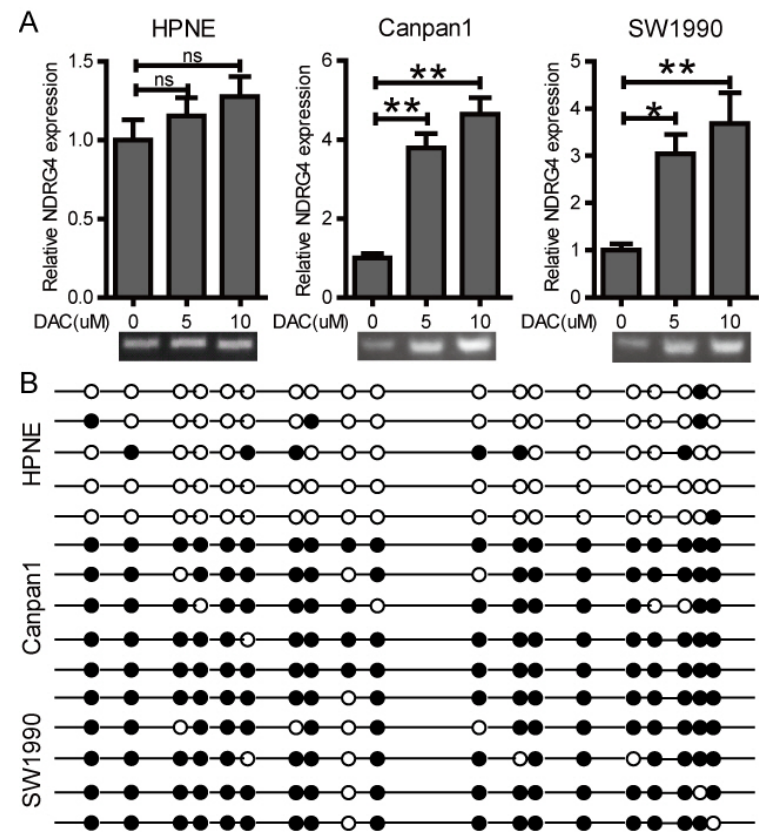

Fig. 2. Down-regulated NDRG4 resulting from hypermethylation of DNA promoter. (A) NDRG4 mRNA expression was detected in PDAC (SW1990 and Canpan1) and HPNE cells treated with no drug, DAC, TSA, or DAC plus TSA. GAPDH was used as a loading control. The upper row shows qRT-PCR data and the lower row shows PCR bands. ${ }^{*} \mathrm{P}<0.05$, $* \mathrm{P}<0.01$, ns $=$ no significance. (B) Hypermethylation of the NDRG4 promoter was detected in SW1990, Canpan1, and HPNE cells by bisulfite-sequencing. Solid circles = methylated CpG site; Open circles = unmethylated CpG site.

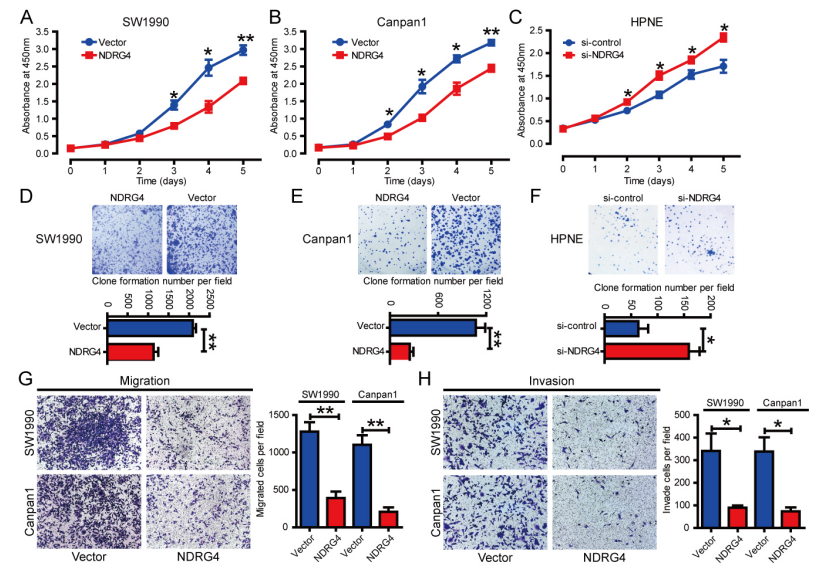

Fig. 3. NDRG4 overexpression inhibits viability and motility of PDAC cells in vitro. (A and B) NDRG4 overexpression significantly decreases proliferation of SW1990 (A) and Canpan1 (B) cells. (C) NDRG4 knockdown significantly increases proliferation of HPNE cells. (D and E) Up-regulated NDRG4 markedly suppresses clone formation ability of SW1990 (D) and Canpan1 (E) cells. (F) NDRG4 knockdown markedly enhances clone formation ability of HPNE cells. (G and H) NDRG4 overexpression reduces migration (E) and invasion $(F)$ of PDAC cells. ${ }^{*} P<0.05, * * P<0.01$.

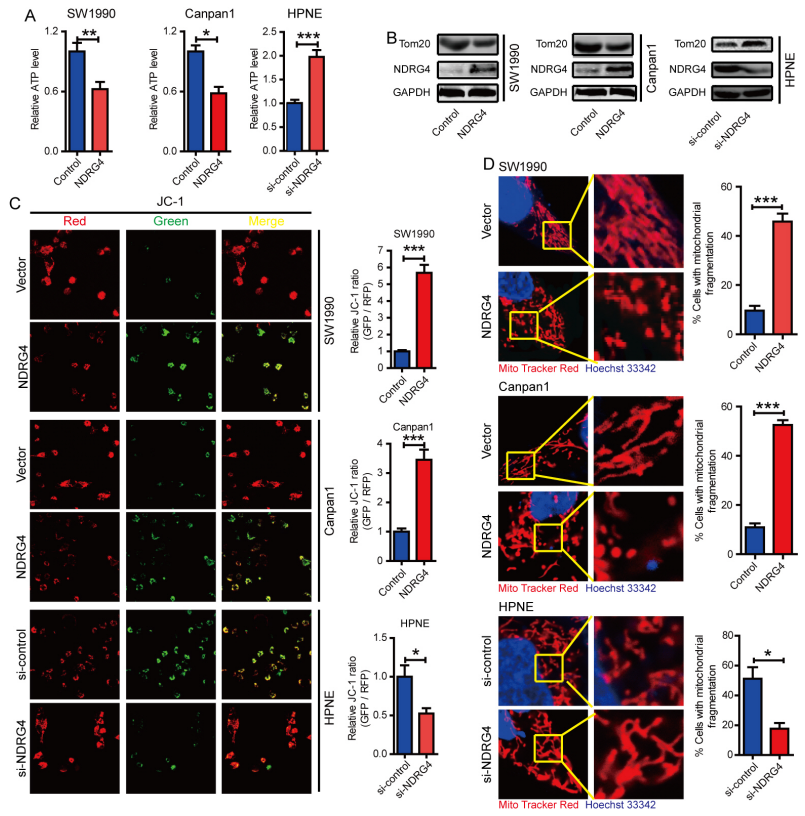

Fig. 4. NDRG4 overexpression regulates mitochondrial function. (A) NDRG4 overexpression leads to decreased ATP production in SW1990 and Canpan 1 cells, Opposite results were obtained for HPNE cells with NDRG4 knockdown. (B) Tom20 was detected in SW1990, Canpan1, and HPNE cells with NDRG4 overexpression or knockdown. (C) Confocal images of mitochondrial membrane potential (MMP) treated with NDRG4 overexpression or knockdown in SW1990, Canpan1, and HPNE cells. Cells were stained with JC-1. The right histogram for quantified MMP was given as JC-1 ratio (GFP/RFP). (D) Confocal images of mitochondrial morphology treated with NDRG4 overexpression or knockdown in SW1990, Canpan1, and HPNE cells. Cells were stained with Mito-Tracker Red (red) and Hoechst33342 (blue). The right histogram reflects the degree of mitochondrial morphological change in cells $(n=100)$.

obtained for HPNE cells treated with NDRG4 knockdown (Fig. 3C and 3F). Next, we investigated whether NDRG4 could affect the motility of PDAC cells. We found that overexpression of NDRG4 significantly suppressed the migration (Fig. 3G) and invasion (Fig. 3H) of PDAC cells (SW1990 and Canpan1). These data suggest that NDRG4 is involved in malignant properties of tumor cells.

\section{NDRG4 mediates mitochondrial function in PDAC cells}

Data from genecards (https://www.genecards.org/) and immunofluorescence showed that NDRG4 was located in the cytoplasm and the mitochondria (Supplementary Fig. 2). In view of this, we hypothesized that NDRG4 expression might regulate mitochondrial function. To investigate whether NDRG4 expression might regulate ATP level, we performed ATP assay. Results showed that NDRG4 overexpression resulted in decreased ATP production in SW1990 and Canpan1 cells (Fig. 4A). Meanwhile, NDRG4 overexpression weakened expression levels of a mitochondrial marker (Fig. 4B). We then analyzed mitochondrial membrane potential using a JC-1 fluorescent probe. Results showed that 
GFP/RFP ratio was significantly increased in up-regulated NDRG4 cells than in control cells (Fig. 4C). In line with this finding, fluorescence microscopy labeled by MitoTracker ${ }^{\circledR}$ Red revealed that NDRG4 overexpression in SW1990 and Canpan1 cells induced a significant increase in the number of fragmented mitochondria with decreases of elongated, reticular, and intermediate mitochondria (Fig. 4D). However, opposite results were obtained for HPNE cells after NDRG4 knockdown (Fig. 4A-4D). These results strongly highlight the significance of NDRG4 expression in mediating the mitochondrial function of PDAC cells.

\section{DISCUSSION}

Recently, NDRG family proteins have attracted attention because some studies have reported that NDRG family proteins are important for tumorigenesis and tumor progression $(5,6)$. Studies on the role of NDRG4 in cancer are gaining more and more attention. However, NDRG4 has received little attention in PDAC. In the beginning, we detected a relationship between mRNA expression of NDRG4 and prognosis of patients with PDAC. Results showed that abnormal NDRG4 expression was associated with patients' outcome. Meanwhile, low expression of NDRG4 was found in GEO datasets and PDAC cells, consistent with its expression pattern in breast cancer (15), colon cancer (18), and gastric cancer. (14) These data suggest that NDRG4 also works as a suppressor in PDAC. However, more studies need to be performed to detect protein expression levels of NDRG4 in patient samples.

Hyper-methylation of $\mathrm{CpG}$ islands in the promoter region of suppressor is considered to be an important inducer of cancer progression, including colorectal cancer (19), gastric cancer (20), esophageal cancer (21), and PDAC (22). Recent reports have revealed that epigenetic mechanisms are particularly important in controlling NDRG4 expression in cancer, including colorectal cancer (18), gastric cancer (17), and breast cancer (15). The genomic structure of NDRG4 by online analysis showed a dense CpG island near the transcription initiation site. Meanwhile, reactivation of NDRG4 was induced after treatment with DNA methyltransferase. Bisulfite sequencing also confirmed hypermethylation in the promoter region of NDRG4. More importantly, we firstly reported that NDRG4 was epigenetic silenced by hypermethylation of its promoter in PDAC, consistent with previous reports about its role in other pathological processes $(15,17,18)$. Therefore, reactivating NDRG4 expression with a demethylation agent might have potential value for PDAC therapy. This is worthy of further in-depth study. Previously, CpG island methylation level of NDRG4 promoter has been validated to be a promised early detection marker for colorectal cancer $(18,23,24)$. In USA, NDRG4 methylation has been approved as one molecular marker for multi-target stool DNA test by the FDA. It is currently used as a screening modality (24). Hence, further studies should be carried out to detect the relationship of NDRG4 methylation with clinical parameters and prognosis using samples such as tissue and pancreatic juice of patients with different cancer stages.

Our results provided evidence that NDRG4 exerted as a tumor suppressor in PDAC. Overexpression of NDRG4 in PDAC cells inhibited cell proliferation and invasion in vitro. This suggests that NDRG4 is a tumor suppressor in PDAC. Interestingly, the localization of NDRG4 in the mitochondria drove us to clarify its role in mitochondrial function. Results showed that NDRG4 overexpression could weaken the mitochondrial function in PDAC cells, including decreased ATP production, increased depolarization, and fragmented mitochondria. Meanwhile, NDRG4 overexpression weakened expression levels of Tom20, a mitochondrial marker. Previously studies have illuminated the relationship between mitochondrial function and cell function, including proliferation, apoptosis, cell differentiation, and so on (25-27). Therefore, we speculate that NDRG4 can lead to the loss of mitochondrial membrane potential and induce mitochondrial division to promote mitochondrial quality control, which in turn will induce mitochondrial autophagy and reduce the number of mitochondria. It might even induce the activation of mitochondrial apoptosis pathway. Further detailed research studies are needed to clarify antecedents and consequences of the effect of NDRG4 on mitochondrial function.

In summary, our study provides the first evidence to describe a tumor suppressor role for NDRG4 in PDAC. This is the first report demonstrating that hypermethylation-driven NDRG4 silencing can promote PDAC by regulating mitochondrial function. It will advance our understanding of the role of NDRG4 in tumor progression.

\section{MATERIALS AND METHODS}

\section{Data mining}

Online analysis was performed using Kaplan-Meier plotter (http:// kmplot.com/analysis/) and oncolnc (http://www.oncolnc.org/). Hazard ratio (and 95\% confidence intervals) and log-rank $\mathrm{P}$ were calculated. Two GEO datasets (GSE28735 and GSE62452) were found at https://www.ncbi.nlm.nih.gov/geo.

\section{Cell culture and cell transduction}

Human PDAC cell lines (PANC1, Canpan1, SW1990 and ASPC1) and normal human pancreatic duct cells (HPNE) were obtained from Shanghai Institute of Biochemistry and Cell Biology (Shanghai, China). They were cultured in DMED medium supplemented with $10 \%$ FBS at $37^{\circ} \mathrm{C}$ with $5 \% \mathrm{CO}_{2}$. SW1990 and Canpan1 cells were infected with lentivirus (lentiviru-NDRG4 or empty vehicle control) in the presence of $8 \mu \mathrm{g} / \mathrm{ml}$ polybrene (Sigma) and selected with $2 \mu \mathrm{g} / \mathrm{ml}$ puromycin (Sangon, Shanghai) for 14 days. NDRG4 stable expression in the selected cell line was verified by western blot.

\section{CCK8 assay}

PDAC cells were seeded into a 96-well plate $(100 \mu \mathrm{l}$ per well) at a density of $5 \times 10^{3}$ cells per well. At indicated end points, 
$10 \mu \mathrm{l}$ of Cell Counting Kit-8 (CCK-8, WST-8, Dojindo, Japan) was added to each well and the absorbance was measured at $450 \mathrm{~nm}$ using a microplate reader. The experiment was performed in triplicate and repeated three times.

\section{Colony-forming assays}

Cells were seeded into a six-well plate (500 cells/well) and cultured in complete medium for two weeks. Cells were fixed with $2 \%$ paraformaldehyde for 30 minutes and stained with $0.1 \%$ crystal violet for another 30 minutes.

\section{In vitro migration and invasion assays}

To determine migration and invasive abilities of PDAC cells with altered expression of NDRG4, transwell assays were conducted. For the migration assay, $4 \times 10^{4}$ cells in $200 \mu \mathrm{l}$ serumfree medium were seeded into the upper chamber (Millicell) of the transwell. The lower chamber was filled with $700 \mu$ l of medium containing $20 \%$ FBS. After $24 \mathrm{~h}$, cells were fixed with $4 \%$ paraformaldehyde for $15 \mathrm{~min}$ and stained with $0.1 \%(\mathrm{w} / \mathrm{v})$ crystal violet for $15 \mathrm{~min}$. The membrane of the upper chamber was cleaned with PBS and air-dried. Images were taken in five random fields using an inverted microscope (Olympus Corp. Tokyo, Japan). The number of cells in each field was counted. For the invasion assay, a similar approach was used. The only difference was that $100 \mu \mathrm{l}$ matrigel (BD Bioscience, Franklin Lakes, NJ) was added into the top chamber of the transwell and $8 \times 10^{4}$ cells were seeded onto the matrigel and cultured for $48 h$.

\section{Mitochondrial morphometrics}

Mitochondrial morphometrics assays were performed as previously reported (28). Mitochondrial staining was carried out with MitoTracker ${ }^{\mathrm{R}}$ Red (Invitrogen) according to the manufacturer's instruction. Images were acquired under a $100 \times$ oil objective using a laser scanning confocal microscope (LSM710, Leica).

\section{Western blot analysis}

Western blot analyses were performed as described previously (29). Primary antibodies used were those against NDRG4 (1 : 1,000; Proteintech), Tom20 (1: 1,000; Abcam), and GAPDH (1: 5,000, Proteintech). After incubating with IRDye 680 anti-mouse IgG (LI-COR, Lincoln, NE) and IRDye 800 anti-rabbit IgG (LI-COR, Lincoln, NE) secondary antibodies, signal intensities were analyzed using an Odyssey Infrared Image System (LI-COR Biosciences).

\section{DAC treatment and quantitative real-time PCR}

Cells were treated with 5-aza-2'-deoxycytidine (DAC, SigmaAldrich, St. Louis, MO, USA) or same volume of Dimethyl Sulfoxide (DMSO) for 3 days. At the indicated end point, total RNA was extracted from cells using Trizol reagent (Takara, Dalian, China). qPCR was performed as described previously (30). Primers used were as follows: NDRG4-F: 5'-GGCCTTCTGCATG
TAGTGATCCG-3', NDRG4-R: 5'-GGTGATCTCCTGCATGTCC TCG-3'; GAPDH-F: 5'-GTCAACGGATTTGGTCTGTATT-3', and GAPDH-R: 5'-AGTCTTCTGGGTGGCAGTGAT-3'.

\section{DNA methylation analysis}

DNA methylation assay was performed as previously reported (31). Briefly, DNA (1 $\mu \mathrm{g})$ was bisulfite modified according to the manufacturer's instruction (Epitect Bisulfite Kit, Qiagen) and amplified with designed sequence-specific primers for binding to the NDRG4 promoter: $5^{\prime}$-TTAGAGGTTTTTGAGTTTTT GGTTTT-3' (forward) and 5'-CCCTCCAAACCCCCTATAAC-3' (reverse).

\section{ATP assay}

An enhanced ATP assay kit (Beyotime, Shanghai, China) was used to determine ATP concentrations according to the manufacture's protocol.

\section{Mitochondrial membrane potential (JC-1)}

Assays were performed using a JC-1 kit (Beyotime, Shanghai, China) according to the manufacture's protocol. Fluorescence labeled cells were imaged with a fluorescence microscope and analyzed with an EnSpire Reader. The ratio of fluorescence at $530 \mathrm{~nm}$ to that at $590 \mathrm{~nm}$ emission was used for measuring mitochondrial membrane potential.

\section{Statistical analysis}

Data are presented as means \pm SD of three independent experiments. All statistical analyses were performed using Student's t-test. P-value of less than 0.05 was considered as statistically significant.

\section{ACKNOWLEDGEMENTS}

We appreciate all authors for their assistance with experiments and constructive comments for the manuscript.

\section{CONFLICTS OF INTEREST}

The authors have no conflicting interests.

\section{REFERENCES}

1. Kamisawa T, Wood LD, Itoi T and Takaori K (2016) Pancreatic cancer. Lancet 388, 73-85

2. Wolfgang CL, Herman JM, Laheru DA et al (2013) Recent progress in pancreatic cancer. CA Cancer J Clin 63, 318-348

3. Miller KD, Siegel RL, Lin CC et al (2016) Cancer treatment and survivorship statistics, 2016. CA Cancer J Clin 66, 271-289

4. Chen W, Zheng R, Baade PD et al (2016) Cancer statistics in China, 2015. CA Cancer J Clin 66, 115-132

5. Zhou RH, Kokame K, Tsukamoto Y, Yutani C, Kato H and Miyata T (2001) Characterization of the human NDRG 
gene family: a newly identified member, NDRG4, is specifically expressed in brain and heart. Genomics 73, 86-97

6. Melotte V, Qu X, Ongenaert M et al (2010) The N-myc downstream regulated gene (NDRG) family: diverse functions, multiple applications. FASEB J 24, 4153-4166

7. Okuda T, Kokame K and Miyata T (2008) Differential expression patterns of NDRG family proteins in the central nervous system. J Histochem Cytochem 56, 175-182

8. Ohki T, Hongo S, Nakada N, Maeda A and Takeda M (2002) Inhibition of neurite outgrowth by reduced level of NDRG4 protein in antisense transfected PC12 cells. Brain Res Dev Brain Res 135, 55-63

9. Qu X, Jia H, Garrity DM et al (2008) Ndrg4 is required for normal myocyte proliferation during early cardiac development in zebrafish. Dev Biol 317, 486-496

10. Fontenas L, De Santis F, Di Donato V, Degerny C and Chambraud B (2016) Neuronal Ndrg4 is essential for nodes of Ranvier organization in zebrafish. PLoS Genet 12, e1006459

11. Dupays L, Kotecha S, Angst B and Mohun TJ (2009) Tbx2 misexpression impairs deployment of second heart field derived progenitor cells to the arterial pole of the embryonic heart. Dev Biol 333, 121-131

12. Schilling $\mathrm{SH}$, Hjelmeland $\mathrm{AB}$, Radiloff $\mathrm{DR}$ et al (2009) NDRG4 is required for cell cycle progression and survival in glioblastoma cells. J Biol Chem 284, 25160-25169

13. Kotipatruni RP, Ren X, Thotala D and Jaboin JJ (2015) NDRG4 is a novel oncogenic protein and p53 associated regulator of apoptosis in malignant meningioma cells. Oncotarget 6, 17594-17604

14. Zhang Z, She J, Yang J et al (2018) NDRG4 in gastric cancer determines tumor cell proliferation and clinical outcome. Mol Carcinog 57, 762-771

15. Jandrey EHF, Moura RP, Andrade LNS et al (2019) NDRG4 promoter hypermethylation is a mechanistic biomarker associated with metastatic progression in breast cancer patients. NPJ Breast Cancer 5, 11

16. Chu D, Zhang Z, Zhou $Y$ et al (2015) NDRG4, a novel candidate tumor suppressor, is a predictor of overall survival of colorectal cancer patients. Oncotarget 6, 7584-7596

17. Chen X, Yang Y, Liu J et al (2017) NDRG4 hypermethylation is a potential biomarker for diagnosis and prognosis of gastric cancer in Chinese population. Oncotarget 8, 8105-8119

18. Melotte V, Lentjes MH, van den Bosch SM et al (2009) $\mathrm{N}-\mathrm{Myc}$ downstream-regulated gene 4 (NDRG4): a candi- date tumor suppressor gene and potential biomarker for colorectal cancer. J Natl Cancer Inst 101, 916-927

19. Okugawa Y, Grady WM and Goel A (2015) Epigenetic alterations in colorectal cancer: emerging biomarkers. Gastroenterology 149, 1204-1225.e1212

20. Tahara T and Arisawa T (2015) DNA methylation as a molecular biomarker in gastric cancer. Epigenomics 7, 475-486

21. Zheng YZ, Wen J, Cao X et al (2015) Decreased mRNA expression of transcription factor forkhead box $F 2$ is an indicator of poor prognosis in patients with resected esophageal squamous cell carcinoma. Mol Clin Oncol 3, 713719

22. Liu B and Pilarsky C (2018) Analysis of DNA hypermethylation in pancreatic cancer using methylation-specific PCR and bisulfite sequencing. Methods Mol Biol 1856, 269-282

23. Ahlquist DA, Zou H, Domanico M et al (2012) Nextgeneration stool DNA test accurately detects colorectal cancer and large adenomas. Gastroenterology 142, 248-256; quiz e225-246

24. Imperiale TF, Ransohoff DF, Itzkowitz SH et al (2014) Multitarget stool DNA testing for colorectal-cancer screening. N Engl J Med 370, 1287-1297

25. Trotta AP and Chipuk JE (2017) Mitochondrial dynamics as regulators of cancer biology. Cell Mol Life Sci 74, 1999-2017

26. Youle RJ and van der Bliek AM (2012) Mitochondrial fission, fusion, and stress. Science 337, 1062-1065

27. Pradhan RK, Qi F, Beard DA and Dash RK (2010) Characterization of membrane potential dependency of mitochondrial $\mathrm{Ca} 2+$ uptake by an improved biophysical model of mitochondrial Ca2 + uniporter. PLoS One 5, e13278

28. Xie KF, Guo DD and Luo XJ (2019) SMDT1-driven change in mitochondrial dynamics mediate cell apoptosis in PDAC. Biochem Biophys Res Commun 511, 323-329

29. Chakraborty PK, Mustafi SB, Xiong X et al (2017) MICU1 drives glycolysis and chemoresistance in ovarian cancer. Nat Commun 8, 14634

30. Bustin SA, Benes V, Garson JA et al (2009) The MIQE guidelines: minimum information for publication of quantitative real-time PCR experiments. Clin Chem 55, 611-622

31. Fu Y, Feng MX, Yu J et al (2014) DNA methylationmediated silencing of matricellular protein dermatopontin promotes hepatocellular carcinoma metastasis by $\alpha 3 \beta 1$ integrin-Rho GTPase signaling. Oncotarget 5, 6701-6715 